\title{
Millimeterwave Spectral Studies of Propynal (HCCCHO) Produced by DC Glow Discharge and Ab Initio DFT Calculation
}

\author{
A. I. Jaman, ${ }^{1}$ Rangana Bhattacharya, ${ }^{1}$ Debasish Mandal, ${ }^{2}$ and Abhijit K. Das ${ }^{2}$ \\ ${ }^{1}$ Experimental Condensed Matter Physics Division, Saha Institute of Nuclear Physics, Sector-1, Block-AF, Bidhannagar, \\ Kolkata 700 064, India \\ ${ }^{2}$ Department of Spectroscopy, Indian Association for the Cultivation of Science, Jadavpur, Kolkata 700 032, India
}

Correspondence should be addressed to A. I. Jaman, aismail.jaman@saha.ac.in

Received 25 July 2011; Revised 30 September 2011; Accepted 5 October 2011

Academic Editor: George C. King

Copyright () 2011 A. I. Jaman et al. This is an open access article distributed under the Creative Commons Attribution License, which permits unrestricted use, distribution, and reproduction in any medium, provided the original work is properly cited.

\begin{abstract}
The ground-state $(v=0)$ millimeterwave rotational spectrum of propynal (HCCCHO) produced by a DC glow discharge through a low-pressure $\left(\sim 10-20\right.$ mTorr) flow of propargyl alcohol $\left(\mathrm{HCCCH}_{2} \mathrm{OH}\right)$ vapor has been observed in the frequency region: 36.0-94.0 GHz. Measured rotational transition frequencies along with the previously reported microwave and millimeterwave transitions were fitted to a standard asymmetric-top Hamiltonian to determine an improved set of rotational and centrifugal distortion constant. Detailed DFT calculations were also carried out with various functional and basis sets to evaluate the spectroscopic constants, dipole moment, and various structural parameters of propynal and compared with the corresponding experimental values.
\end{abstract}

\section{Introduction}

Propynal (HCCCHO) also known as propiolic aldehyde has been found in interstellar molecular clouds TMC-1 and Sgr B2(N-LMH) [1-3]. In 1955 Howe and Goldstein [4] reported the $J=2 \leftarrow 1$ and $J=3 \leftarrow 2$ microwave transitions of the most abundant isotopic species of propynal. They have determined the dipole moment of the molecule to be $\mu=$ $2.46 \pm 0.04$ D. Later on, Costain and Morton [5] determined the rotational constants $B, C$, and $\left(A-D_{k}\right)$ for the parent as well as various isotopic species and were able to determine an accurate substitution structure. Winnewisser [6] has extended the analysis of the ground state of propynal to the millimeterwave region $(95.0-200.0 \mathrm{GHz})$ which has yielded a set of ground state rotational and centrifugal distortion constants. Reference [6] includes a set of predicted $a$-type Rbranch frequencies from 37.0 to $94.0 \mathrm{GHz}$ covering $J=4 \leftarrow$ 3 to $J=10 \leftarrow 9$ transitions which are of prime importance to radio astronomers for conducting search for this molecule in the interstellar space. Infrared-microwave double-resonance spectroscopic study of propynal using a Zeeman tuned HeXe laser as the infrared source was conducted by Takami and Shimoda [7] to determine the rotational constants of the $v_{2}=1$ excited vibrational state. Later on, Jones [8] has reported the rotational constants of propynal in the ground as well as several excited state combination and hot bands using a $\mathrm{CO}_{2}$ laser as the infrared source. In all the previous works propynal was either procured commercially or prepared chemically.

Production of new molecules, molecular ions, and radicals by applying a DC glow discharge through a low-pressure flow of gas or a mixture of gases inside an absorption cell has proved to be an efficient and useful technique [9]. Recently, Jaman [10] has reported an analysis of the millimeterwave rotational spectra of propyne $\left(\mathrm{CH}_{3} \mathrm{CCH}\right)$ produced by a DC glow discharge through a mixture of toluene and acetylene vapor and carried out a detailed DFT calculation to evaluate the spectroscopic constants and molecular parameters and compared them with experimental values. In the present communication, we report the analysis of the ground-state $(v=0)$ rotational spectra of propynal produced by a DC glow discharge through a low-pressure flow of propargyl alcohol $\left(\mathrm{HCCCH}_{2} \mathrm{OH}\right)$ vapor in the frequency region 36.094.0 GHz. Asymmetric-top $K_{-1} K_{+1}$-structures of different $J+1 \leftarrow J$ transitions which fall under this frequency range have been observed and measured. The measured 


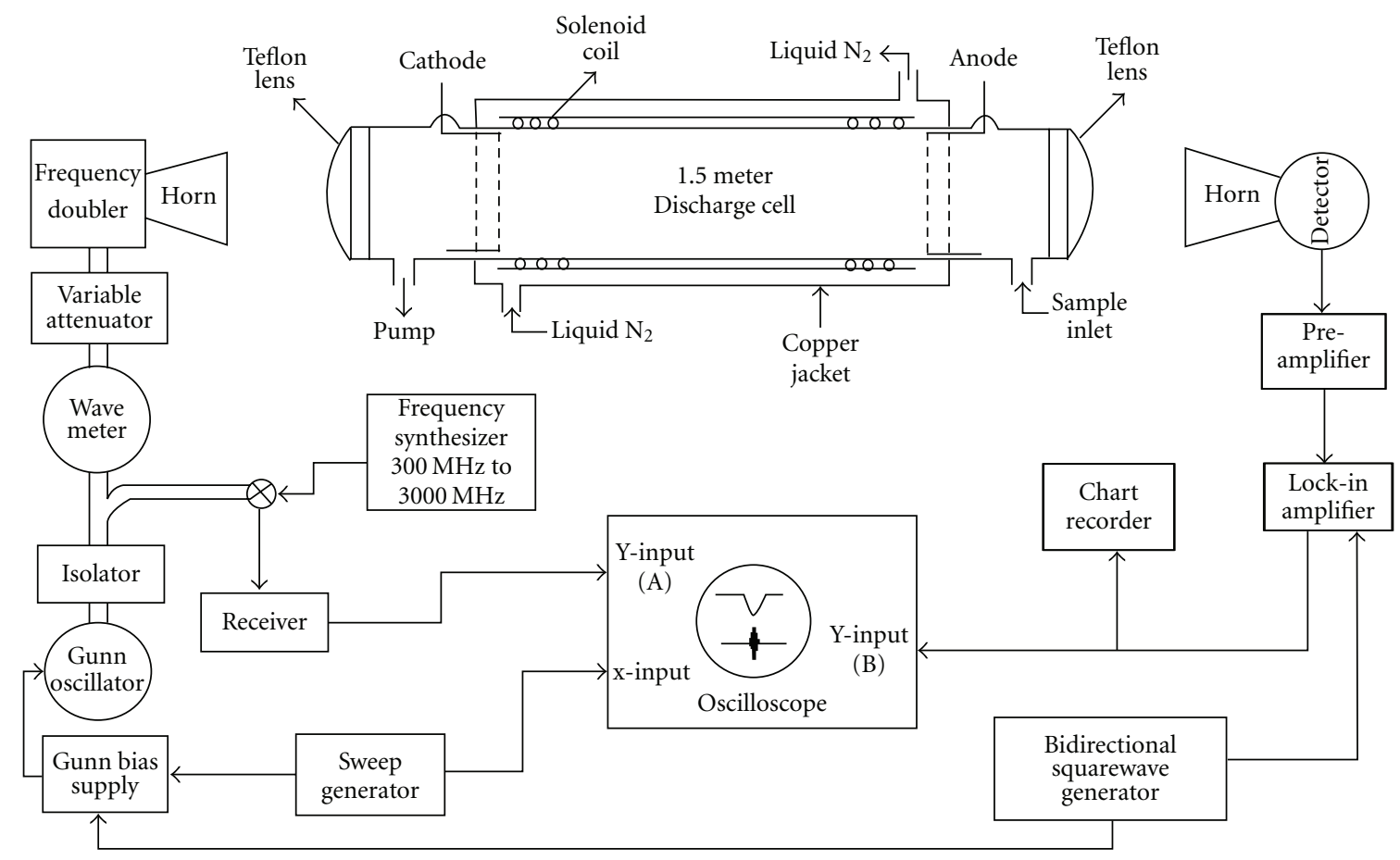

FIGURE 1: Block diagram of source modulated millimeterwave spectrometer with DC discharge facility.

rotational transition frequencies along with the previously reported frequencies were fitted to standard asymmetric-top Hamiltonian to determine an improved set of rotational and centrifugal distortion (CD) constants. A detailed quantum chemical calculation was also carried out to evaluate the spectroscopic constants, dipole moment, and the structural parameters of propynal. Finally, the experimentally determined rotational and $\mathrm{CD}$ constants were compared with the best set of values obtained after a series of DFT calculations. Work on the analysis of the rotational spectrum of propenal $\left(\mathrm{CH}_{2} \mathrm{CHCHO}\right)$ produced by DC glow discharge is underway.

\section{Experimental Details}

The spectrometer used in the present work is basically a $50 \mathrm{kHz}$ source-modulated system combined with a free space glass discharge cell of $1.5 \mathrm{~m}$ in length and $10 \mathrm{~cm}$ in diameter. The cell is fitted with two Teflon lenses at each end. A high-voltage DC regulated power supply $(6 \mathrm{kV}, 1300 \mathrm{~mA})$ procured from Glassman, Japan was used to apply a DC voltage through a flow of low-pressure precursor gases. The cell is connected with a high vacuum pump at one end and to the sample holder section through a glass port on the other.

Klystrons and Gunn diodes followed by frequency doubler (Millitech model MUD-15-H23F0 and MUD-10-LF000) have been used as radiation sources. Millimeterwave radiation was fed into the cell by a waveguide horn and Teflon lens. A similar horn and lens arrangement was used to focus the millimeterwave power onto the detector after propagating through the cell. The output frequency of the millimeterwave radiation was frequency modulated by a bidirectional squarewave of $50 \mathrm{kHz}$ [11], and the signal from the detector (Millitech model DBT-15-RP000 and DXP-10RPFW0) was amplified by a $100 \mathrm{kHz}$ tuned preamplifier and detected by a phase-sensitive lock-in amplifier in the $2 \mathrm{f}$ mode. The output of the lock-in amplifier was connected to an oscilloscope or a chart recorder for signal display. The uncertainty in frequency measurement has been estimated to be $\pm 0.15 \mathrm{MHz}$. A block diagram of the spectrometer is shown in Figure 1. Details of the spectrometer used have been described elsewhere $[12,13]$.

Propynal was produced inside the absorption cell by applying a DC glow discharge through a low-pressure $(\sim 5-$ 10 mTorr) flow of propargyl alcohol $\left(\mathrm{HCCCH}_{2} \mathrm{OH}\right)$ vapor. The discharge current was maintained at around $5 \mathrm{~mA}$ with an applied voltage of $1.0 \mathrm{kV}$. A mechanical on/off type discharge was found to be suitable to observe good signals of propynal. A controlled flow of liquid nitrogen vapor was used to cool the cell during the experiment. The observed signals of propynal appeared as sharp lines immediately after the DC discharge was applied but started losing intensity with time. Application of fresh discharge helps in regaining the previous intensity.

\section{Computational Method}

Quantum chemical computations were performed using GAUSSIAN 03 package [14]. Density functional methods with various functionals were used to calculate the structural parameters, dipole moment, and total energy (sum of electronic and zero point energy) as well as the rotational and centrifugal distortion constants of propynal. The geometry 
TABLE 1: Microwave and millimeterwave rotational transition frequencies of propynal (HCCCHO) in the ground vibrational state (in MHz.).

\begin{tabular}{|c|c|c|c|c|c|c|c|}
\hline \multicolumn{6}{|c|}{ Transition } & \multicolumn{2}{|c|}{ Frequency } \\
\hline$J^{\prime}$ & $K_{-1}^{\prime}$ & $K_{+1}^{\prime}$ & $J^{\prime \prime}$ & $K_{-1}^{\prime \prime}$ & $K_{+1}^{\prime \prime}$ & Obs. Freq. & Obs-Calc. \\
\hline 2 & 0 & 2 & 1 & 0 & 1 & 18650.33 & .03 \\
\hline 2 & 1 & 2 & 1 & 1 & 1 & 18325.56 & .03 \\
\hline 2 & 1 & 1 & 1 & 1 & 0 & 18978.78 & .00 \\
\hline 2 & 1 & 2 & 3 & 0 & 3 & 34903.64 & .25 \\
\hline 3 & 0 & 3 & 2 & 0 & 2 & 27972.13 & -.05 \\
\hline 3 & 1 & 2 & 2 & 1 & 1 & 28467.15 & -.10 \\
\hline 3 & 1 & 3 & 2 & 1 & 2 & 27487.48 & .06 \\
\hline 3 & 2 & 1 & 2 & 2 & 0 & 27985.53 & -.30 \\
\hline 3 & 2 & 2 & 2 & 2 & 1 & 27980.86 & .08 \\
\hline 3 & 1 & 3 & 4 & 0 & 4 & 25100.65 & -.01 \\
\hline 4 & 1 & 4 & 5 & 0 & 5 & 15146.06 & .02 \\
\hline 4 & 0 & 4 & 3 & 0 & 3 & $37290.20^{\mathrm{a}}$ & .06 \\
\hline 4 & 1 & 4 & 3 & 1 & 3 & $36648.20^{\mathrm{a}}$ & -.05 \\
\hline 4 & 1 & 3 & 3 & 1 & 2 & $37954.60^{\mathrm{a}}$ & .00 \\
\hline 4 & 2 & 3 & 3 & 2 & 2 & $37306.50^{\mathrm{a}}$ & -.02 \\
\hline 4 & 2 & 2 & 3 & 2 & 1 & $37319.20^{\mathrm{a}}$ & .06 \\
\hline 5 & 0 & 5 & 4 & 0 & 4 & $46602.90^{\mathrm{a}}$ & .02 \\
\hline 5 & 1 & 4 & 5 & 0 & 5 & $65852.60^{\mathrm{a}}$ & .04 \\
\hline 5 & 1 & 5 & 4 & 1 & 4 & $45807.70^{\mathrm{a}}$ & .01 \\
\hline 5 & 1 & 4 & 4 & 1 & 3 & $47440.40^{\mathrm{a}}$ & -.06 \\
\hline 5 & 2 & 4 & 4 & 2 & 3 & $46631.30^{\mathrm{a}}$ & .07 \\
\hline 5 & 2 & 3 & 4 & 2 & 2 & $46656.50^{\mathrm{a}}$ & .04 \\
\hline 5 & 3 & 2 & 4 & 3 & 1 & $46645.80^{\mathrm{a}}$ & .01 \\
\hline 6 & 0 & 6 & 5 & 0 & 5 & $55909.00^{\mathrm{a}}$ & -.09 \\
\hline 6 & 1 & 5 & 5 & 1 & 4 & $56924.40^{\mathrm{a}}$ & -.04 \\
\hline 6 & 1 & 6 & 5 & 1 & 5 & $54965.40^{\mathrm{a}}$ & .00 \\
\hline 6 & 2 & 5 & 5 & 2 & 4 & $55954.60^{\mathrm{a}}$ & -.07 \\
\hline 6 & 1 & 5 & 6 & 0 & 6 & $66868.00^{\mathrm{a}}$ & .09 \\
\hline 7 & 4 & 3 & 6 & 4 & 2 & $65317.60^{\mathrm{a}}$ & .14 \\
\hline 7 & 5 & 2 & 6 & 5 & 1 & $65334.00^{\mathrm{a}}$ & -.23 \\
\hline 7 & 0 & 7 & 6 & 0 & 6 & $65207.60^{\mathrm{a}}$ & .11 \\
\hline 7 & 1 & 6 & 6 & 1 & 5 & $66406.10^{\mathrm{a}}$ & -.06 \\
\hline 7 & 1 & 6 & 7 & 0 & 7 & $68066.60^{\mathrm{a}}$ & .02 \\
\hline 7 & 2 & 6 & 6 & 2 & 5 & $65276.40^{\mathrm{a}}$ & -.18 \\
\hline 7 & 2 & 5 & 6 & 2 & 4 & $65347.10^{\mathrm{a}}$ & -.05 \\
\hline 7 & 3 & 4 & 6 & 3 & 3 & $65307.30^{\mathrm{a}}$ & -.10 \\
\hline 8 & 1 & 7 & 8 & 0 & 8 & $69455.00^{\mathrm{a}}$ & .02 \\
\hline 8 & 1 & 8 & 7 & 1 & 7 & $73274.30^{\mathrm{a}}$ & -.03 \\
\hline 9 & 0 & 9 & 8 & 0 & 8 & $83775.80^{\mathrm{a}}$ & -.02 \\
\hline 9 & 1 & 9 & 8 & 1 & 8 & $82425.00^{\mathrm{a}}$ & .08 \\
\hline 9 & 1 & 8 & 8 & 1 & 7 & $85361.10^{\mathrm{a}}$ & -.09 \\
\hline 9 & 1 & 8 & 9 & 0 & 9 & $71040.30^{\mathrm{a}}$ & -.05 \\
\hline 9 & 2 & 8 & 8 & 2 & 7 & $83914.90^{\mathrm{a}}$ & .10 \\
\hline 9 & 2 & 7 & 8 & 2 & 6 & $84065.75^{\mathrm{a}}$ & .04 \\
\hline 9 & 3 & 7 & 8 & 3 & 6 & $83971.10^{\mathrm{a}}$ & .01 \\
\hline 9 & 3 & 6 & 8 & 3 & 5 & $83972.50^{a}$ & .00 \\
\hline 9 & 4 & 6 & 8 & 4 & 5 & $83981.50^{\mathrm{a}}$ & .06 \\
\hline
\end{tabular}


Table 1: Continued.

\begin{tabular}{|c|c|c|c|c|c|c|c|}
\hline \multicolumn{6}{|c|}{ Transition } & \multicolumn{2}{|c|}{ Frequency } \\
\hline 9 & 5 & 4 & 8 & 5 & 3 & $84001.50^{\mathrm{a}}$ & .01 \\
\hline 9 & 6 & 3 & 8 & 6 & 2 & $84028.60^{\mathrm{a}}$ & -.03 \\
\hline 9 & 7 & 2 & 8 & 7 & 1 & $84061.80^{\mathrm{a}}$ & -.05 \\
\hline 10 & 0 & 10 & 9 & 1 & 9 & $36693.10^{\mathrm{a}}$ & .02 \\
\hline 10 & 1 & 9 & 10 & 0 & 10 & $72830.60^{\mathrm{a}}$ & -.13 \\
\hline 10 & 1 & 10 & 9 & 1 & 9 & $91572.50^{\mathrm{a}}$ & .01 \\
\hline 10 & 0 & 10 & 9 & 0 & 9 & $93043.30^{\mathrm{a}}$ & -.03 \\
\hline 10 & 1 & 9 & 9 & 1 & 8 & $94833.70^{\mathrm{a}}$ & .03 \\
\hline 10 & 2 & 9 & 9 & 2 & 8 & $93230.60^{\mathrm{a}}$ & .00 \\
\hline 10 & 2 & 8 & 9 & 2 & 7 & $93437.80^{\mathrm{a}}$ & .03 \\
\hline 10 & 3 & 8 & 9 & 3 & 7 & $93304.40^{\mathrm{a}}$ & .06 \\
\hline 10 & 3 & 7 & 9 & 3 & 6 & $93306.80^{\mathrm{a}}$ & .05 \\
\hline 10 & 4 & 7 & 9 & 4 & 6 & $93313.90^{\mathrm{a}}$ & -.03 \\
\hline 11 & 0 & 11 & 10 & 0 & 10 & 102298.00 & -.04 \\
\hline 11 & 0 & 11 & 10 & 1 & 10 & 47418.60 & -.02 \\
\hline 11 & 1 & 11 & 10 & 1 & 10 & 100716.90 & .06 \\
\hline 11 & 1 & 10 & 10 & 1 & 9 & 104302.20 & -.06 \\
\hline 11 & 2 & 10 & 10 & 2 & 9 & 102543.90 & .08 \\
\hline 11 & 2 & 9 & 10 & 2 & 8 & 102819.60 & .03 \\
\hline 11 & 3 & 9 & 10 & 3 & 8 & 102638.50 & .05 \\
\hline 11 & 3 & 8 & 10 & 3 & 7 & 102642.30 & -.04 \\
\hline 11 & 4 & 8 & 10 & 4 & 7 & 102646.80 & -.04 \\
\hline 11 & 5 & 7 & 10 & 5 & 6 & 102669.10 & .07 \\
\hline 11 & 6 & 6 & 10 & 6 & 5 & 102701.00 & .06 \\
\hline 11 & 7 & 5 & 10 & 7 & 4 & 102740.90 & .09 \\
\hline 11 & 8 & 4 & 10 & 8 & 3 & 102788.00 & .13 \\
\hline 11 & 9 & 3 & 10 & 9 & 2 & 102841.80 & .14 \\
\hline 12 & 0 & 12 & 11 & 0 & 11 & 111539.10 & .07 \\
\hline 12 & 0 & 12 & 11 & 1 & 11 & 58240.80 & .00 \\
\hline 12 & 1 & 12 & 11 & 1 & 11 & 109857.70 & .01 \\
\hline 12 & 1 & 11 & 11 & 1 & 10 & 113766.50 & .11 \\
\hline 12 & 2 & 11 & 11 & 2 & 10 & 111854.30 & -.06 \\
\hline 12 & 2 & 10 & 11 & 2 & 9 & 112211.90 & .09 \\
\hline 12 & 3 & 10 & 11 & 3 & 9 & 111973.40 & -.06 \\
\hline 12 & 3 & 9 & 11 & 3 & 8 & 111979.50 & -.07 \\
\hline 12 & 4 & 9 & 11 & 4 & 8 & 111980.00 & -.06 \\
\hline 12 & 5 & 8 & 11 & 5 & 7 & 112002.80 & .00 \\
\hline 12 & 6 & 7 & 11 & 6 & 6 & 112036.90 & -.03 \\
\hline 12 & 7 & 6 & 11 & 7 & 5 & 112080.00 & .03 \\
\hline 12 & 8 & 5 & 11 & 8 & 4 & 112131.00 & .00 \\
\hline 12 & 8 & 4 & 11 & 8 & 3 & 112131.00 & .00 \\
\hline 12 & 9 & 4 & 11 & 9 & 3 & 112189.40 & -.11 \\
\hline 12 & 9 & 3 & 11 & 9 & 2 & 112189.40 & -.11 \\
\hline 13 & 0 & 13 & 12 & 1 & 12 & 69148.40 & .06 \\
\hline 14 & 1 & 13 & 14 & 0 & 14 & 82226.80 & .01 \\
\hline 15 & 1 & 14 & 15 & 0 & 15 & 85185.00 & -.06 \\
\hline 16 & 0 & 16 & 15 & 0 & 15 & 148346.60 & .09 \\
\hline 19 & 0 & 19 & 18 & 0 & 18 & 175770.30 & -.02 \\
\hline 19 & 1 & 19 & 18 & 1 & 18 & 173725.80 & .06 \\
\hline
\end{tabular}


Table 1: Continued.

\begin{tabular}{|c|c|c|c|c|c|c|c|}
\hline \multicolumn{6}{|c|}{ Transition } & \multicolumn{2}{|c|}{ Frequency } \\
\hline 19 & 1 & 18 & 18 & 1 & 17 & 179851.70 & -.01 \\
\hline 19 & 2 & 18 & 18 & 2 & 17 & 176928.80 & .11 \\
\hline 19 & 2 & 17 & 18 & 2 & 16 & 178295.50 & .05 \\
\hline 19 & 3 & 17 & 18 & 3 & 16 & 177342.70 & .00 \\
\hline 19 & 3 & 16 & 18 & 3 & 15 & 177403.80 & .02 \\
\hline 19 & 4 & 16 & 18 & 4 & 15 & 177327.50 & .01 \\
\hline 19 & 4 & 15 & 18 & 4 & 14 & 177328.50 & -.03 \\
\hline 19 & 5 & 15 & 18 & 5 & 14 & 177342.30 & -.07 \\
\hline 19 & 6 & 14 & 18 & 6 & 13 & 177384.90 & -.08 \\
\hline 19 & 7 & 13 & 18 & 7 & 12 & 177446.20 & -.04 \\
\hline 19 & 8 & 12 & 18 & 8 & 11 & 177522.60 & -.05 \\
\hline 19 & 9 & 11 & 18 & 9 & 10 & 177612.20 & .01 \\
\hline 19 & 10 & 10 & 18 & 10 & 9 & 177714.10 & -.01 \\
\hline 19 & 11 & 9 & 18 & 11 & 8 & 177827.80 & .10 \\
\hline 19 & 12 & 8 & 18 & 12 & 7 & 177952.80 & .15 \\
\hline 19 & 12 & 7 & 18 & 12 & 6 & 177952.80 & .15 \\
\hline 19 & 14 & 6 & 18 & 14 & 5 & 178235.40 & -.19 \\
\hline 20 & 0 & 20 & 19 & 0 & 19 & 184875.80 & .14 \\
\hline 20 & 1 & 20 & 19 & 1 & 19 & 182830.80 & -.07 \\
\hline 20 & 3 & 18 & 19 & 3 & 17 & 186683.80 & -.14 \\
\hline 20 & 3 & 17 & 19 & 3 & 16 & 186762.80 & .01 \\
\hline 20 & 4 & 16 & 19 & 4 & 15 & 186666.70 & -.06 \\
\hline 20 & 4 & 17 & 19 & 4 & 16 & 186665.20 & -.05 \\
\hline 20 & 5 & 15 & 19 & 5 & 14 & 186676.90 & -.11 \\
\hline 20 & 5 & 16 & 19 & 5 & 15 & 186676.90 & -.09 \\
\hline 20 & 6 & 15 & 19 & 6 & 14 & 186719.70 & -.02 \\
\hline 20 & 6 & 14 & 19 & 6 & 13 & 186719.70 & -.02 \\
\hline 20 & 7 & 14 & 19 & 7 & 13 & 186782.90 & -.03 \\
\hline 20 & 8 & 13 & 19 & 8 & 12 & 186862.50 & -.03 \\
\hline 20 & 9 & 12 & 19 & 9 & 11 & 186956.20 & .01 \\
\hline 20 & 10 & 11 & 19 & 10 & 10 & 187063.10 & .07 \\
\hline 3 & 1 & 3 & 4 & 0 & 4 & 25100.65 & -.01 \\
\hline 4 & 1 & 4 & 5 & 0 & 5 & 15146.06 & .02 \\
\hline 9 & 0 & 9 & 8 & 1 & 8 & 26074.66 & -.05 \\
\hline 16 & 0 & 16 & 15 & 1 & 15 & 102261.30 & .10 \\
\hline 17 & 0 & 17 & 16 & 1 & 16 & 113385.90 & -.09 \\
\hline 20 & 0 & 20 & 19 & 1 & 19 & 146837.00 & .15 \\
\hline 4 & 1 & 4 & 3 & 0 & 3 & 99039.07 & .01 \\
\hline 5 & 1 & 5 & 4 & 0 & 4 & 107556.60 & .00 \\
\hline 6 & 1 & 6 & 5 & 0 & 5 & 115919.10 & -.03 \\
\hline 10 & 1 & 10 & 9 & 0 & 9 & 147922.70 & -.09 \\
\hline 14 & 1 & 14 & 13 & 0 & 13 & 177974.20 & .07 \\
\hline 7 & 2 & 6 & 8 & 1 & 7 & 109566.70 & -.06 \\
\hline 8 & 2 & 6 & 9 & 1 & 9 & 113757.10 & .12 \\
\hline 9 & 2 & 7 & 10 & 1 & 10 & 106250.20 & .00 \\
\hline 10 & 2 & 8 & 11 & 1 & 11 & 98971.03 & -.07 \\
\hline 6 & 4 & 2 & 5 & 4 & 1 & 55985.80 & -.09 \\
\hline 6 & 3 & 3 & 5 & 3 & 2 & 55976.20 & -.03 \\
\hline 6 & 2 & 4 & 5 & 2 & 3 & 55998.90 & .09 \\
\hline
\end{tabular}

${ }^{\mathrm{a}}$ This work, rest are from $[4,6]$. 
TABLE 2: Ground-state rotational and centrifugal distortion constants of propynal (HCCHO).

\begin{tabular}{lccc}
\hline Constants & $\begin{array}{c}\text { "Global” fit using } \\
\text { microwave and } \\
\text { millimeterwave data }\end{array}$ & Reference [6] & $\begin{array}{c}\text { DFT } \\
\text { calculation }\end{array}$ \\
\hline $\mathrm{A}(\mathrm{MHz})$ & $68035.251 \pm 0.021$ & $68035.299 \pm 0.043$ & 69124.41 \\
$B(\mathrm{MHz})$ & $4826.224 \pm 0.001$ & $4826.3014 \pm 0.0073$ & 4816.87 \\
$C(\mathrm{MHz})$ & $4499.588 \pm 0.001$ & $4499.5107 \pm 0.0069$ & 4495.94 \\
$D_{J}(\mathrm{kHz})$ & $1.868 \pm 0.001$ & $1.917 \pm 0.012$ & 1.764 \\
$D_{J K}(\mathrm{kHz})$ & $-147.757 \pm 0.030$ & $-148.102 \pm 0.047$ & -127.969 \\
$D_{K}(\mathrm{kHz})$ & $8978.402 \pm 5.58$ & $8991.240 \pm 9.73$ & 8110.126 \\
$d_{1}(\mathrm{kHz})$ & $-0.353 \pm 0.004$ & $0.3458 \pm 0.0014$ & -0.315 \\
$d_{2}(\mathrm{kHz})$ & $-0.022 \pm 0.002$ & $-19.500 \pm 1.59$ & -0.018 \\
$H_{J K}(\mathrm{~Hz})$ & $-0.659 \pm 0.047$ & $-0.741 \pm 0.059$ & \\
$H_{K J}(\mathrm{~Hz})$ & $-8.336 \pm 0.125$ & $-8.310 \pm 0.130$ & \\
$h_{1}(\mathrm{~Hz})$ & $0.0120 \pm 0.007$ & - & \\
$\sigma^{\mathrm{b}}$ & 0.0814 & 0.08721 & \\
$\kappa^{\mathrm{c}}$ & -0.989 & -0.989 & \\
$\Delta^{\mathrm{d}}$ & 0.173 & 0.177 & \\
$N^{\mathrm{e}}$ & 145 & 96 & \\
\hline
\end{tabular}

${ }^{\mathrm{b}}$ Standard deviation of the overall fit.

${ }^{\mathrm{c}}$ Asymmetry parameter $\kappa=(2 B-A-C) /(A-C)$.

${ }^{\mathrm{d}}$ Inertia defect $\Delta=I_{c}-I_{b}-I_{a}$.

${ }^{\mathrm{e}}$ Number of transitions used in the fit.

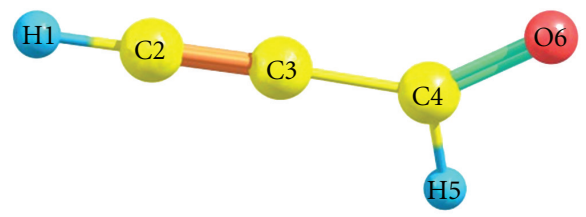

Figure 2: Optimized geometry of propynal molecule and the numbering of atoms.

optimization was carried out using Becke 3-term correlation functional (B3LYP) with basis sets $6-31+g(d, p)$ to $6-311++g$ (d, 2p), Becke three-parameter hybrid functional and Perdew/Wang 91 nonlocal correlation functional (B3PW91) method with different basis sets from 6-31g to 6-311++g(d, $2 \mathrm{p})$, modified Perdew-Wang one-parameter hybrid model taking basis sets from 6-31g to 6-311++g(d, 2p), and Perdew, Burke, and Ernzerhof functional (PBEPBE) with the basis sets $6-311 \mathrm{~g}$ to $6-311++\mathrm{g}(\mathrm{d}, 2 \mathrm{p})$. The frequency calculation was done on optimized geometry to have true minima. The molecular drawing is done by using CHEMCRAFT (trial version) [15].

\section{Rotational Spectrum and Analysis}

The rotational spectrum of propynal was predicted in the frequency range $36.0-94.0 \mathrm{GHz}$ using the rotational and centrifugal distortion constants reported earlier [6]. Accordingly, $J=4 \leftarrow 3$ to $J=10 \leftarrow 9$ transitions along with their different $K_{-1} K_{+1}$ components were predicted and compared

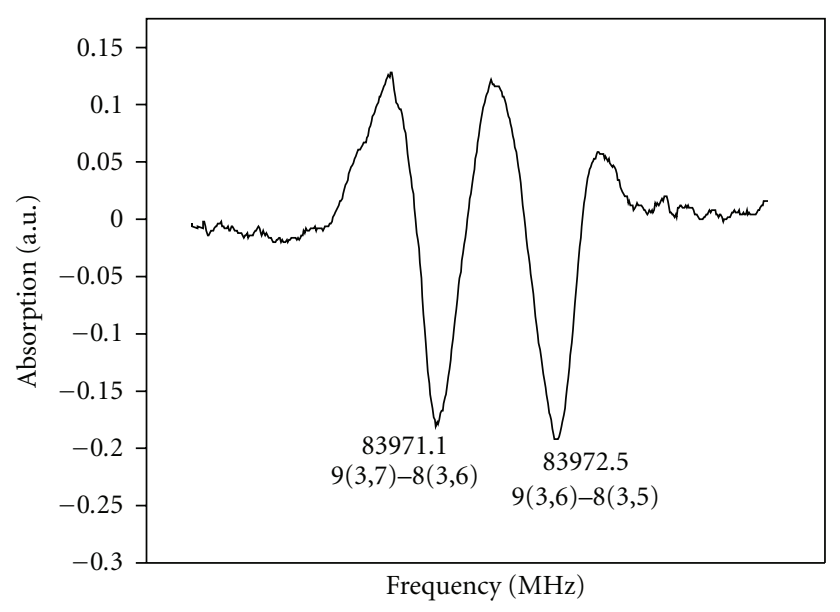

FIgURE 3: Observed trace of the $K_{-1}=3$ doublet of $J=9 \leftarrow 8$ transition of propynal produced by DC discharge.

with some of the predictions given in [6]. Different $K_{-1} K_{+1}$ components in each series were measured. Finally, $145 a$ - and $b$-type R- and Q-branch transitions consisting of all previous microwave [5], millimeterwave [6], and present data were fit to the semirigid rotor Watson's S-reduction Hamiltonian ( $I^{r}$-representation) [16] to determine an improved set of spectroscopic constants including five quartic $\left(D_{J}, D_{J K}\right.$, $D_{K}$, etc.) and three sextic $\left(H_{J K}, H_{K J}\right.$, and $\left.h_{1}\right)$ centrifugal distortion constants. Being a light molecule (mol. wt. 54), the shifts in frequency of the absorption lines from their rigid rotor positions due to centrifugal distortion were found to be substantial for the higher $J$ and $K_{-1}$ transitions. The maximum shift was found to be $1049.55 \mathrm{MHz}$ for $19(14,6)-$ $18(14,5)$ transition. The assigned new transitions and those reported by earlier workers $[5,6]$ are listed in Table 1 . The inclusion of sextic constants $H_{J K}, H_{K J}$, and $h_{1}$ were found to be necessary to reproduce the observed frequencies with high $J$, high $K_{-1}$ millimeterwave transitions. The contribution of the sextic distortion constant $H_{J}$ was found to be too small to be determined. All the ground state spectroscopic constants obtained for propynal using this "global" fit are listed in Table 2. A typographical error for the CD constant $d_{2}\left(R_{5}\right)=-19.500 \pm 1.59 \mathrm{kHz}$ in [6] has been noticed. The value of $d_{2}$ in [6] should have been either $-0.0195 \mathrm{kHz}$ or $-19.500 \mathrm{~Hz}$. The new value of $d_{2}$ in our case comes out to be $-0.022 \pm 0.002 \mathrm{kHz}$. It is quite evident from Table 2 that the standard deviations of most of the parameters have come down with this analysis as compared to their respective values reported in [6]. The agreement between the derived set of spectroscopic constants and those obtained earlier $[5,6]$ with commercial samples indicates that the newly assigned transition frequencies of Table 1 definitely belong to propynal, a discharge product of propargyl alcohol. Figure 2 shows the observed trace of the $K_{-1}=3$ doublet of $J=$ $9 \leftarrow 8$ transition immediately after the DC discharge was applied. The trace remained visible for a couple of minutes on the oscilloscope screen with gradually diminishing intensity. 
TABLE 3: Calculated ground-state rotational constants of propynal (HCCCHO) in various models and basis sets.

\begin{tabular}{|c|c|c|c|c|c|}
\hline & Model & Basis set & $A_{0}(\mathrm{MHz})$ & $B_{0}(\mathrm{MHz})$ & $C_{0}(\mathrm{MHz})$ \\
\hline \multirow{12}{*}{ DFT } & B3LYP & $6-31+g(d, p)$ & 68098.21 & 4784.41 & 4463.15 \\
\hline & B3LYP & $6-31++g(d, 2 p)$ & 68256.89 & 4782.68 & 4462.32 \\
\hline & B3LYP & $6-311++g(d, 2 p)$ & 69124.41 & 4816.87 & 4495.94 \\
\hline & B3PW91 & $6-31 g$ & 66270.21 & 4767.54 & 4440.57 \\
\hline & B3PW91 & $6-31++g(d, 2 p)$ & 68576.97 & 4791.35 & 4471.19 \\
\hline & B3PW91 & $6-311++g(d, 2 p)$ & 69756.37 & 4820.39 & 4501.66 \\
\hline & MPW1PW91 & $6-31 g$ & 66636.15 & 4783.87 & 4456.38 \\
\hline & MPW1PW91 & $6-31++g(d, 2 p)$ & 69083.92 & 4804.28 & 4484.75 \\
\hline & MPW1PW91 & $6-311++g(d, 2 p)$ & 70009.21 & 4833.54 & 4514.27 \\
\hline & PBEPBE & 6-311g & 64622.84 & 4749.88 & 4417.10 \\
\hline & PBEPBE & $6-311++g(d, 2 p)$ & 68197.19 & 4764.26 & 4448.29 \\
\hline & PBE1PBE & $6-31++g(d, 2 p)$ & 64197.26 & 4750.79 & 4417.61 \\
\hline Expt. $^{\mathrm{f}}$ & & & 68035.25 & 4826.22 & 4499.59 \\
\hline
\end{tabular}

${ }^{\mathrm{f}}$ This work.

TABLE 4: Comparison of the molecular optimized geometry, dipole moment, and total energy of propynal calculated by various methods and basis sets with the experimental values.

\begin{tabular}{|c|c|c|c|c|c|c|c|c|c|c|}
\hline Method & Model & Basis set & $\begin{array}{c}1 \mathrm{H}-2 \mathrm{C} \\
(\AA)\end{array}$ & $\begin{array}{c}2 \mathrm{C}-3 \mathrm{C} \\
(\AA)\end{array}$ & $\begin{array}{c}3 \mathrm{C}-4 \mathrm{C} \\
(\AA)\end{array}$ & $\begin{array}{c}4 \mathrm{C}-5 \mathrm{H} \\
(\AA)\end{array}$ & $\begin{array}{c}4 \mathrm{C}-6 \mathrm{O} \\
(\AA)\end{array}$ & $\begin{array}{c}\text { (3C-4C-6O) } \\
\text { (in degree) }\end{array}$ & $\mu(\mathrm{D})$ & Energy (a.u) \\
\hline \multirow{12}{*}{ DFT } & B3LYP & $6-31+g^{* *}$ & 1.059 & 1.212 & 1.452 & 1.114 & 1.219 & 123.89 & 3.141 & -190.6240574 \\
\hline & B3LYP & $6-31++g(d, 2 p)$ & 1.058 & 1.212 & 1.452 & 1.113 & 1.219 & 123.94 & 3.125 & -190.6246385 \\
\hline & B3LYP & $6-311++g(d, 2 p)$ & 1.054 & 1.204 & 1.450 & 1.110 & 1.211 & 124.06 & 3.074 & -190.6720249 \\
\hline & B3PW91 & $6-31 g$ & 1.059 & 1.216 & 1.441 & 1.108 & 1.242 & 124.38 & 3.244 & -190.4741521 \\
\hline & B3PW91 & $6-31++g(d, 2 p)$ & 1.059 & 1.212 & 1.450 & 1.114 & 1.216 & 123.94 & 3.095 & -190.5414422 \\
\hline & B3PW91 & $6-311++g(d, 2 p)$ & 1.056 & 1.204 & 1.448 & 1.112 & 1.209 & 124.09 & 3.038 & -190.5861953 \\
\hline & MPW1PW91 & 6-31g & 1.057 & 1.214 & 1.439 & 1.106 & 1.239 & 124.32 & 3.254 & -190.4964469 \\
\hline & MPW1PW91 & $6-31++g(d, 2 p)$ & 1.058 & 1.209 & 1.449 & 1.111 & 1.213 & 123.90 & 3.095 & -190.565401 \\
\hline & MPW1PW91 & $6-311++g(d, 2 p)$ & 1.055 & 1.202 & 1.448 & 1.110 & 1.206 & 124.06 & 3.042 & -190.609715 \\
\hline & PBEPBE & $6-311 \mathrm{~g}$ & 1.063 & 1.219 & 1.442 & 1.117 & 1.253 & 124.14 & 3.111 & -190.3871779 \\
\hline & PBEPBE & $6-311++g(d, 2 p)$ & 1.062 & 1.216 & 1.449 & 1.123 & 1.223 & 124.10 & 3.002 & -190.4447923 \\
\hline & PBE1PBE & $6-31++g(d, 2 p)$ & 1.063 & 1.221 & 1.441 & 1.117 & 1.254 & 124.06 & 3.284 & -190.3966287 \\
\hline Expt.g & & & 1.055 & 1.208 & 1.444 & 1.106 & 1.215 & 123.80 & $2.747^{\mathrm{h}}$ & \\
\hline
\end{tabular}

${ }^{\mathrm{g}}[5],{ }^{\mathrm{h}}[17]$.

\section{Computational Results}

Propynal is a slightly asymmetric prolate top molecule $(\kappa=-0.989)$. The optimization of geometry for propynal has been tested at various levels of theory and basis sets. However, the computed rotational and centrifugal distortion constants obtained with B3LYP level of theory with 6$311++g(d, 2 p)$ basis set are found to be in good agreement with the observed values. The model B3PW91 with basis set $6-311++g(d, 2 p)$ also provides the values of spectroscopic constants which are close to the experimental values. But in the later model percentage error of constant $A$ is slightly greater than the previous one. Hence we have chosen B3LYP/6-311++g(d, 2p) configuration for comparing different molecular constants with our experimental values. Results obtained with various models and basis sets are shown in Table 3. The calculated values of rotational and centrifugal distortion constants obtained with DFT B3LYP/ $6-311++g(d, 2 p)$ have been compared with the corresponding experimental values in Table 2. The calculated energy for the optimized geometry of propynal is $5188.185 \mathrm{eV}$, and the dipole moment at the optimized geometry is $3.074 \mathrm{D}$. The number and labeling of propynal molecule is shown in Figure 3. Bond lengths and angle have been computed and are shown in Table 4.

\section{Conclusion}

An efficient method of generating propiolic aldehyde (propynal) in the gas phase by applying a DC glow discharge through a low-pressure vapor of propargyl alcohol inside the absorption cell has been presented. The gas-phase rotational 
spectra of propynal produced in this way have been recorded and analyzed in the frequency range 36.0-94.0 GHz. The asymmetric-top $K_{-1} K_{+1}$-components of different transitions having $J$ values 3 to 9 have been measured. The observed transition frequencies along with the previously reported data $[5,6]$ were fitted to a standard asymmetric-top Watson's S-reduction Hamiltonian ( $I^{r}$-representation) to determine an improved set of rotational and centrifugal distortion constants and compared with the previously reported values of propynal obtained using a commercial sample. A good agreement between the two sets of values confirms that propynal is one of the major discharge products of the propargyl alcohol vapor. To compare the experimental results with theory, DFT calculations were performed using various models and basis sets. However, it was found that B3LYP model with $6-311++\mathrm{g}(\mathrm{d}, 2 \mathrm{p})$ basis set produced the best values of rotational and quartic centrifugal distortion constants which are close to the experimental values.

\section{Acknowledgments}

A. I. Jaman would like to thank Mr. A. K. Bhattacharya for his technical assistance during the course of this work. D. Mandal and A. K. Das are thankful to the Annesur Rahaman Center for High Performance Computing, IACS, for providing computational facilities.

\section{References}

[1] W. M. Irvine, R. D. Brown, D. M. Cragg et al., "A new interstellar polyatomic molecule: detection of propynal in the cold cloud TMC-1," The Astrophysical Journal, vol. 335, no. 2, pp. L89-L93, 1988.

[2] B. E. Turner, "A molecular line survey of sagittarius B2 and orion-kl from 70 to $115 \mathrm{GHz}$. II. Analysis of the data," Astrophysical Journal, Supplement Series, vol. 76, no. 2, pp. 617-686, 1991.

[3] J. M. Hollis, P. R. Jewell, F. J. Lovas, A. Remijan, and H. Møllendal, "Green bank telescope detection of new interstellar aldehydes: propenal and propanal," Astrophysical Journal, vol. 610, no. 1, pp. L21-L24, 2004.

[4] J. A. Howe and J. H. Goldstein, "Microwave spectrum of propiolic aldehyde," The Journal of Chemical Physics, vol. 23, no. 7, pp. 1223-1225, 1955.

[5] C. C. Costain and J. R. Morton, "Microwave spectrum and structure of propynal," The Journal of Chemical Physics, vol. 31, no. 2, pp. 389-393, 1959.

[6] G. Winnewisser, "The ground state of propynal," Journal of Molecular Spectroscopy, vol. 46, no. 1, pp. 16-24, 1973.

[7] M. Takami and K. Shimoda, "Infrared-microwave double resonance of propynal by using the $3.51 \mu \mathrm{m}$ HeXe laser," Journal of Molecular Spectroscopy, vol. 59, no. 1, pp. 35-42, 1976.

[8] H. Jones, "Infrared-microwave double-resonance and twophoton experiments on propynal with $\mathrm{CO}_{2}$ lasers," Journal of Molecular Spectroscopy, vol. 81, no. 1, pp. 21-36, 1980.

[9] S. Saito, "Laboratory microwave spectroscopy of interstellar molecules," Applied Spectroscopy Reviews, vol. 25, no. 3-4, pp. 261-296, 1990.

[10] A. I. Jaman, P. Hemant Kumar, and P. R. Bangal, "Rotational spectrum of Propyne observed in a DC glow discharge and $a b$ initio DFT calculations," Asian Journal of Spectroscopy, pp. 43$48,2010$.

[11] J. F. Verdieck and C. D. Cornwell, "Radio-frequency spectrometer with bidirectional square wave frequency modulation," Review of Scientific Instruments, vol. 32, no. 12, pp. 1383-1386, 1961.

[12] A. I. Jaman, "Millimeterwave spectroscopy of transient molecules produced in a DC discharge," Pramana-Journal of Physics, vol. 61, no. 1, pp. 85-91, 2003.

[13] A. I. Jaman, "Millimeter wave spectrum of ICN, a transient molecule of chemical and astrophysical interest," Journal of Physics: Conference Series, vol. 80, no. 1, Article ID 012006, 2007.

[14] Gaussian 03, Revision-B.03, Gaussian, Pittsburgh, Pa, USA, 2003.

[15] http://www.chemcraftprog.com/.

[16] J. K. G. Watson, "Aspects of quartic and sextic centrifugal effects on rotational energy levels," in Vibrational Spectra and Structure, J. R. Durig, Ed., vol. 6, pp. 1-89, Mercel Dekker, New York, NY, USA, 1977.

[17] R. D. Brown and P. D. Godfry, "Stark effect measurements of molecular dipole moments," Australian Journal of Chemistry, vol. 37, no. 9, pp. 1951-1954, 1984. 

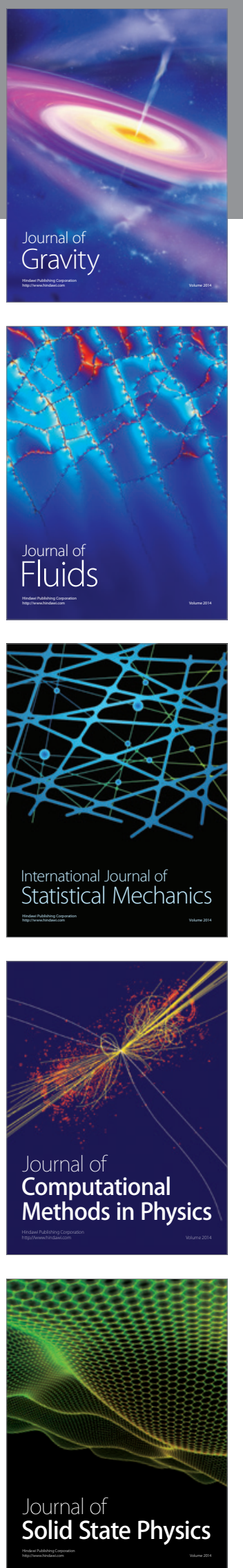

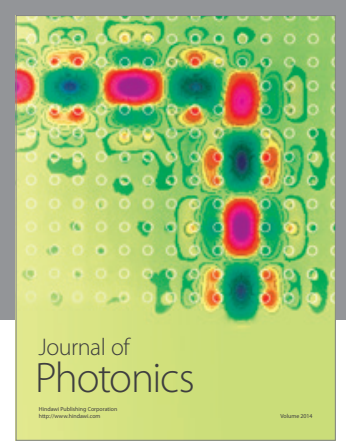

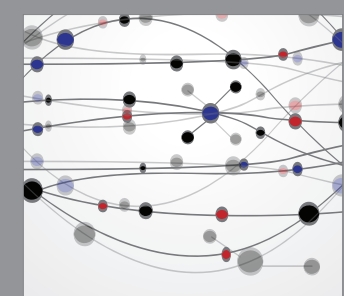

The Scientific World Journal
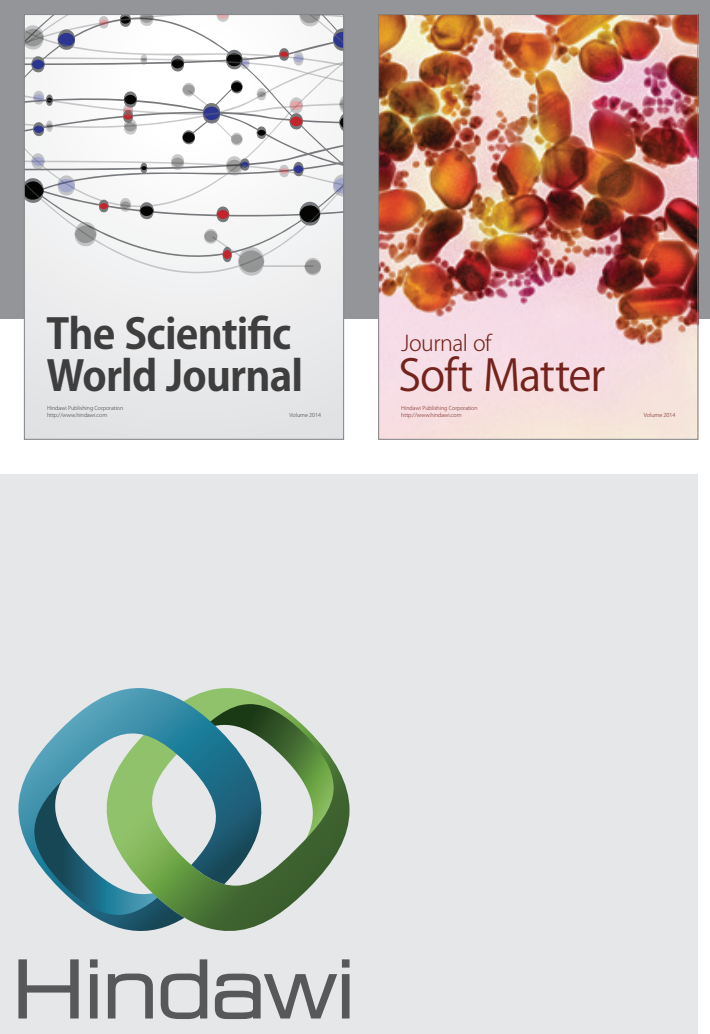

Submit your manuscripts at

http://www.hindawi.com
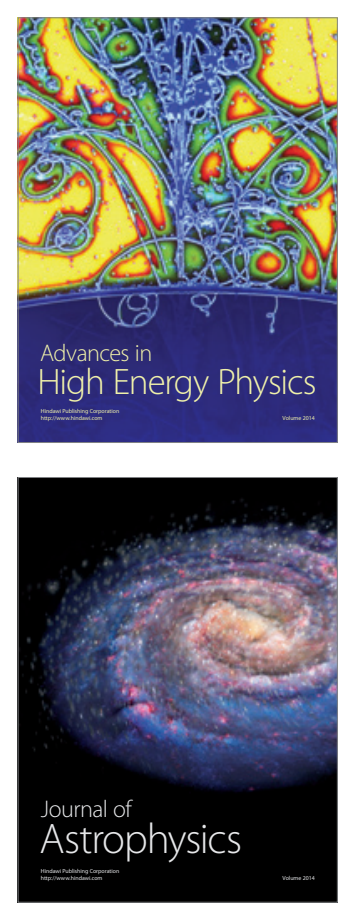
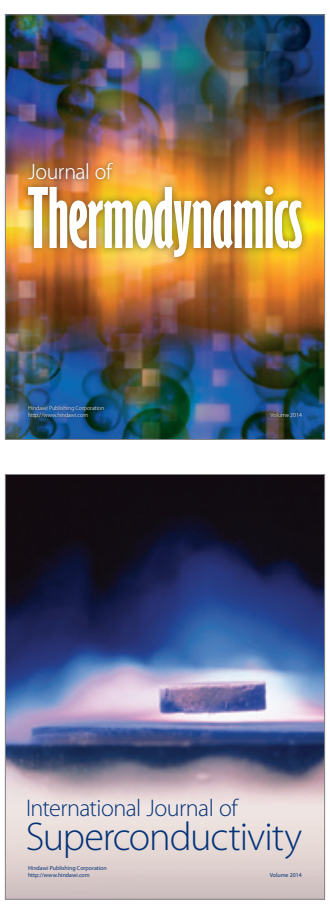
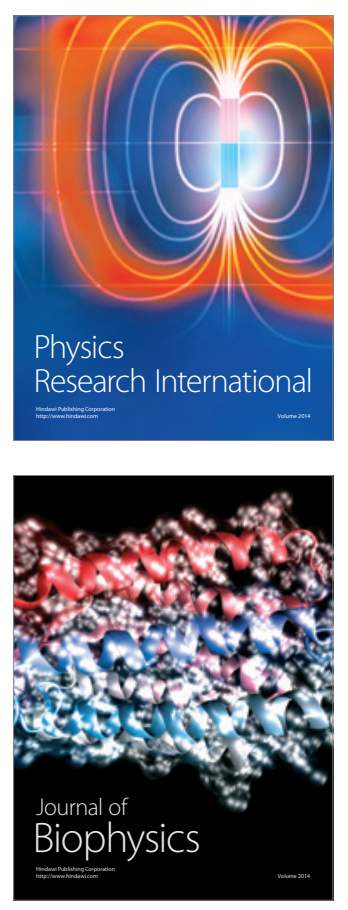
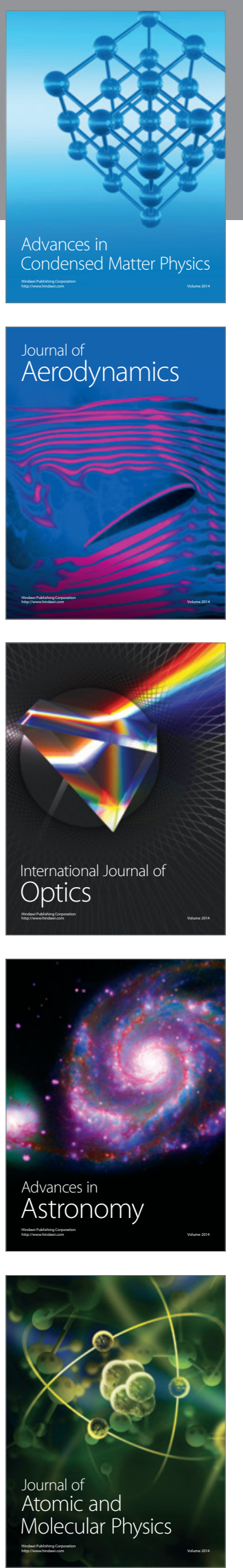